\title{
Pattern Classification of Melanoma by Local Features Using BoF Based Spatial Encoding
}

\author{
Felsia Thompson \\ a Research Scholar is with the department of Computer Applications, \\ Noorul Islam University, Kumaracoil, India \\ E-Mail: felsiashanu@gmail.com \\ M.K Jeyakumar \\ a Professor is with the department of Computer Applications, \\ Noorul Islam University, Kumaracoil, India \\ E-Mail: jeyakumarmk@yahoo.com
}

\begin{abstract}
Melanoma is the direst form of skin cancer. Early detection of cancer is a very critical issue in today's dematologic practice. Feature extraction allows representing the content of the image as perfectly as possible. In this paper, Bag of Features based Supervised Spatial Encoding of Feature extraction is proposed. Low Level Images and their Latent Features induce codebook of features. Scale invariant Speeded up Robust Features (SURF) technique is used for feature point detection in Low Level Image representation. Since the system considers the entire image as lesion, it also recognizes clustered or patched lesion. It uses $\mathbf{l}^{*} \mathbf{a}^{*} \mathrm{~b}$ color space for describing color intensities. The patterns so detected are classified using multi-SVM classifier. The proposed cluster based system provides the classification accuracy of $95.075 \%$ and sensitivity, specificity rates as $94.07 \%$ and 95.5 respectively.
\end{abstract}

Index Terms- Bag of Features (BoF), dermoscopy, melanoma, Supervised Spatial Encoding(SSE), texture analysis

\section{INTRODUCTION}

MELANOMA also known as malignant melanoma is a type of cancer that develops from the pigmentcontaining cells known as melanocytes. In women they most commonly occur on the legs, while in men they are most common on the back. Sometimes they develop from a mole with concerning changes including an increase in size, irregular edges, change in color, itchiness, or skin breakdown. So, there is a need to detect lesion at an early stage [2]. The pigmented lesions are diagnosed by dermatologists using the technique called dermoscopy. Dermoscopy is a non-invasive diagnosis technique for the in vivo observation of pigmented skin lesions used in dermatology [3]. The standard rules of diagnosis for melanoma are used in dermatology such as the ABCD rule, diagnosis is made subjectively by doctors [5]

Skin cancers are of three types namely: Basal-Cell Cancer (BCC), Squamous-Cell Cancer (SCC) and melanoma [1]. When melanoma occurs, it affects the melanocytic cells thereby increasing the synthesis of melanin. The disorder is characterized by skin lesion development and it varies in shape, size, color and texture [4]. Skin Cancer Detection System is the system to identify and recognize skin cancer symptoms and diagnose melanoma in early stages by analyzing feature variations [6]. Pattern analysis, considered as the classic approach for diagnosis in dermoscopic images [7]. The main textural patterns appeared on the melanocytic lesion are: Globular pattern, Cobblestone pattern, Reticular pattern, Homogeneous pattern, parallel pattern, Starburst pattern, and Multicomponent pattern [25]. They are associated with the predominant local features: Globular pattern with globules, Cobblestone pattern with globules, Reticular pattern with pigment network, Homogeneous pattern with pigmentation, Parallel pattern with furrows and ridges, Starburst pattern with streak, and Multicomponent pattern with a combination of three or more above patterns [8].

The main aim of the paper is to classify these types of lesions by considering the patterns as cluster. The reason behind this decision is that some lesion images come in clusters. The paper represents the images into Low level images. They are extracted by Bag of Features (BoF) representation using SURF technique. Single Scale Spatial Encoding of the above approach provides better recognition results compared with other clustering techniques. Supervised Spatial Encoder (SSE) encodes spatial interaction among the regions in a latent space. This space is induced by codebook of visual features. Latent features of individual regions are then combined to form imagelevel representation by pooling[21]. Multi-SVM classifier then classifies the patterns that are one of the learning techniques used for pattern classification. It analyses and recognizes the image and data patterns used in image processing. The advantage of SVM is that in high dimensional spaces it works effectively and also it uses a subset of training points in the decision function provide memory efficient technique. 


\section{RELATED WORKS}

There are several systems for the identification of melanoma in dermoscopy images. Most of the papers focus to identify the lesion and its malignancy. Papers that focus pattern classification use global methods to classify the pattern. Global methods consider color, shape and texture features [8]-[12]. The standard approach in automatic dermoscopic image analysis has usually three stages: 1) image segmentation; 2) feature extraction and feature selection; and 3) lesion classification.

Yogendra et.al.,[14] presented A new approach for skin cancer classification by which it detect features of a digital image by decomposing the images into different frequency sub bands using wavelet transform. The classification methodology is based on probabilistic neural network and clustering classifier and also classify that whether the given input image is cancerous or non cancerous.

Barata et.al . [15] presented a bag of features approach for the classification of melanomas in dermoscopy images a comparison between color and texture feature. The feature extraction is represented by a two dimensional matrix known as GLCM. The color features are computed by color histograms. The classification is based on K-nearest neighbor and Support Vector Machine (SVM) and the result shows that color feature outperforms the texture feature.

Tanaka et al. [17] presented Pattern classification of nevus with Texture Analysis by considering Co-occurrence matrix on images. Melanoma here is diagnosed by features of specific shape, color and texture. It classifies three patterns by texture analysis: homogeneous pattern, globular pattern and reticular patterns. The tumors are not properly extracted in some images because the area of the tumor was very small and also the contour of the tumor was blurred.

Lucia et.al. [16] presented a novel hierarchical classification system. The segmentation is done by using levelset framework. The texture features are extracted from Generalized Co-occurrence Matrix (GCM). The classification methodology is based on K-NN classifier and it classifies the dermoscopy image into melanoma.

Aurora saez et.al. [18] presented Model based methods of classification of global patterns in dermoscopic images. The segmentation is done by using level-set algorithm. The texture features are extracted by Markov Random Field (MRF).The classification methodology is based on Gaussian model, Gaussian mixture model and bag-of-features model to classify a pigmented lesion into three categories: globular, homogeneous and reticular. The BoF approach use MRF features of local patches sampled from the training set and the overlapping patches are extracted from an image.

This paper uses clustering based melanoma pattern recognition system using spatial encoding with BoF approach for feature extraction. It considers the whole image into Low Level Image (LLI) representation . They are done by BOF feature selection by SURF for that is denoted as $\varphi(x)$. Then convert into vector space by encoding. Region based Statistical Region Merging (SRM) technique [22] [23] is used for segmenting the lesion from the skin .It uses Multi-SVM for pattern classification.

The organization of this paper is as follows: Section II describes aim and objectives of the paper. In Section III, the proposed system overview is discussed. Section IV discusses the methodology adopted. The experimental results are evaluated in Section V and Section VI concludes the result and expresses its future scope.

\section{RESEARCH AIM AND OBJECTIVES}

The purpose of the study is to design and develop a system on melanoma pattern recognition area that focuses on early detection of the malignancy level and patterns associated with skin cancers cells and also have less dependency on medical experts or dermatologists.

The objective of the paper includes:

i) To develop an algorithm for the pattern classification of melanoma by local features using BoF based spatial encoding.

ii) Scale and rotation invariant algorithm also solves the recognition of clustered lesion problem

iii) To ease and early treatment of skin cancer, the system proposes the melanoma pattern recognition system to identify the distribution of patterns at an earlier stage and its pattern type.

\section{SYSTEM OVERVIEW}

The analysis and classification of cluster patterns on melanocytic skin lesion is an object recognition system with specific lesion properties. Given an input image, the system first extracts Low Level Image (LLI) using SURF [24] for the whole image lesion i.e) represent the whole image into BoF representation that is denoted as $\varphi(x)$. Then convert into vector space by encoding. Convert whole image into $\mathrm{N} \times \mathrm{N}$ low level image and $\mathrm{n} \times \mathrm{n}$ window size. Embedding BoF scattered spatial features into embedded feature set. Classifier used here is MultiSVM. The following figure depicts the overall design of the BoF based Spatial encoding. The logical steps of the algorithm as follows: 
Input : Lesion

Output: Melanoma pattern

Step 1 : Read Training database

Step 2: covert image from rgb color space to lab color space.

Step 3: Apply PCA filter for noise removal

Step 4: Apply SURF feature point detection to the whole image for LLI extraction and coding.

Step 5: Pooling and Embedding feature Vectors

Step 6: Read the test image and goto step 1

Step 7: Apply Multi-class SVM for classifying the patterns of the melanoma

\section{METHODOLOGY}

Differentiating the melanoma patterns from the skin lesion needs effective preprocessing, segmentation, feature extraction and classification techniques. This system mainly focuses on Texture analysis phase.

\section{A. Pre-processing}

Image pre-processing is an essential step for dermoscopic images which eliminates noises, leads to increasing accuracy level of the pattern recognition system. Thus, it requires some preprocessing techniques for image enhancement and restoration. Since color information plays a significant role in the analysis of dermoscopic image, the input image here in RGB color space is directly transformed into a LAB color space. And also has the following advantages: (i) decoupling luminance and chromaticity information, and (ii) Achieving invariance to different imaging conditions such as viewing direction, illumination intensity [19]. The advantage of using Lab color space is that it yields uniform color space since Lab is perceptually linear and also the luminance factor $\mathrm{L}$ for all pixels is constant. This will reduce the data size and computation time. The second step is to remove the artifacts from the image. This has been achieved by applying PCA filter on the image database followed by image expansion using contour dilation technique. PCA is used for dimensionality reduction of color and texture space [20].

\section{B. Melanoma Pattern observation by Local features using BoF based Spatial encoding}

As the main aim of the paper is to classify the clustered lesion into patterns, it focuses the entire region. The reason behind this decision is that some lesion images come in clusters. The paper represents images into Low level images. They are extracted by Bag of Features $(\mathrm{BoF})$ representation using SURF techniques. Single scale spatial encoding of the above approach provides better recognition results compared with other clustering technique. Supervised Spatial Encoding of feature analysis framework is shown in the Fig 1.

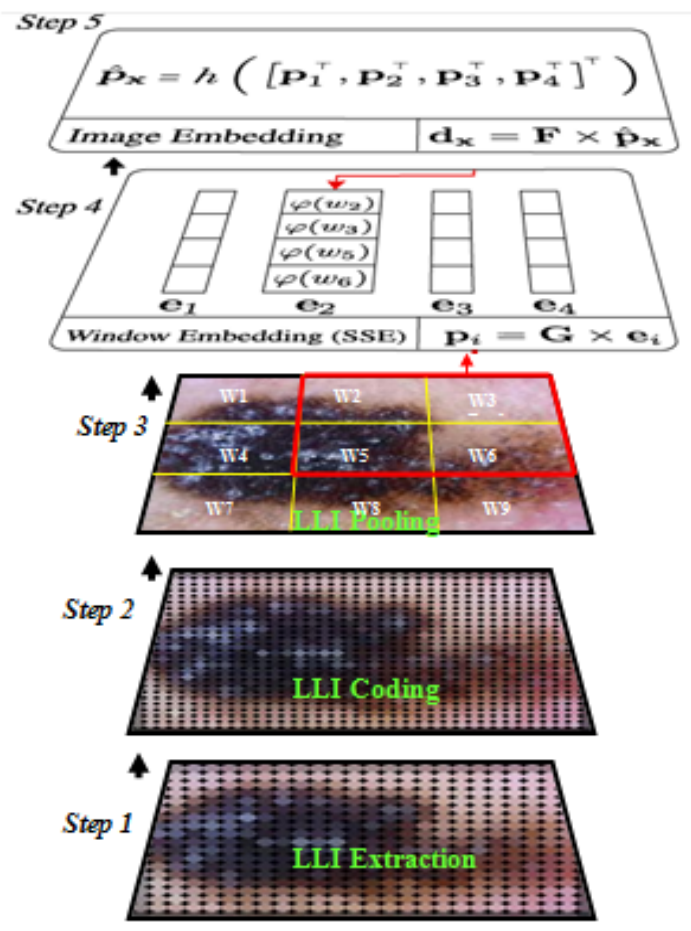

Fig 1. Supervised Spatial Encoder for Melanoma Feature Extraction 


\section{Step 1: Low Level image (LLI) Extraction}

The first step is the low level image extraction. Let $\mathrm{L}$ be the total number of training images $\mathrm{L}=305$ for our system. $\mathrm{Y}$ denotes the class labels $\mathrm{Y}=1,2 \ldots \ldots$. $\mathrm{Y}=4$ for our system. $\mathrm{X}$ is the collection of labeled image denoted as $\left\{x_{i} y_{i}\right\} \mathrm{i}=1,2 \ldots . \mathrm{L}$. Given an image $\mathrm{x}$, partition the whole image into NXN sub regions and extract low level image (LLI) descriptor using SURF for the whole image lesion. i.e) represents the whole image into BoF representation that is denoted as $\varphi(X)$. This system uses single scale.

\section{Step 2: Coding}

The second phase is the feature coding. A coding step encodes each LLI via non-linear feature mapping into vector space. This phase is induced by codebook of image features.

\section{Step 3: Pooling}

The third step is the feature pooling, It concatenates the feature codes into a single feature vector. Let the lesion $\mathrm{X}$ into $\mathrm{N} \times \mathrm{N}$ low level image regions and the window Size be $\mathrm{n} \times n$ regions in M-dimensional latent space. Here $\mathrm{N}=5$ and $\mathrm{n}=2$. For $i^{\text {th }}$ sliding window consisting of $\mathrm{k}=n^{2}$ regions $\left\{w_{a 1}, w_{a 2} \ldots \ldots w_{a k}\right\}$ represents BoF of sliding window regions, vector $e_{i}$ denotes concatenation of its feature vector (FV) as:

$$
e i=\left[\varphi\left(w_{a 1}\right)^{T}, \varphi\left(w_{a 2}\right)^{T} \ldots \ldots \varphi\left(w_{a k}\right)^{T}\right]^{T}
$$

Where $\varphi(w) \in \mathbb{R}^{|\mathcal{D}|}$ is the BoF representation of region $\mathrm{w}$.

\section{Step 4: Window Embedding}

The fourth step is the window embedding. The M-Dimensional Embedding $p_{i}$ for window the $i^{\text {th }}$ window is

$$
P_{i}=G \times e_{i}
$$

where $\mathrm{G} \in \mathbb{R}^{M \times n^{2}} .|\mathcal{D}|$ and taking concatenation of all gives $P_{x}$

\section{Step 5: Image Embedding}

The fifth step is the image embedding. It concatenates latent embedding of all sliding windows into a single vector $P_{x}$ defined as:

$$
P_{x}=\mathrm{h}\left(\left[P_{1}^{T}, P_{2}{ }^{T}, \ldots \ldots \ldots \ldots P_{N^{2}}{ }^{T}\right]\right)
$$

Where $\mathrm{h}()=.\tanh (.)^{1}$. Then the final image-level latent representation $d_{x} \in \mathbb{R}^{M}$ is the result of the second projection

$$
\varphi(x) \equiv d_{x}=F \times P_{x} \quad \text { (4) where } \mathrm{F} \in \mathbb{R}^{M \times N . M} .
$$

\section{Classification}

Even if the analysts were using number of classification techniques for melanoma prediction like ANN classifier, Ada-boost classifier, K-NN classifier and SVM classifier, the experiments show better results for SVM classifier. Since the pattern includes Homogeneous, Globular, Reticular and Multi-Component, the paper uses multi-class SVM for classification.

\section{EXPERIMENTAL RESUlts AND EVALUATION}

\section{A. Experimental data}

The experiment is carried out with 305 dermoscopic images taken from Interactive dermatology atlas, opticomdataresearch of four classes (Globular, Homogenous, Reticular and Multi-component patterns ) is $\mathrm{n}=4$. So each image is divided into 9 sub regions and 4 possible window sliding regions. Initially, SURF based representation considers the Gaussian pyramid octave is 8 that selects strongest features. Upright or rotated feature flag is set to recognize rotated images. The system chooses randomly chosen SURF reticular, multicomponent). The images are in JPEG formats and the images are resized into 256x256 pixels. The system is validated by leave one out approach.-

For evaluating the results, MATLAB R2012a software was used and sample data inputs as follows in Fig 2. 

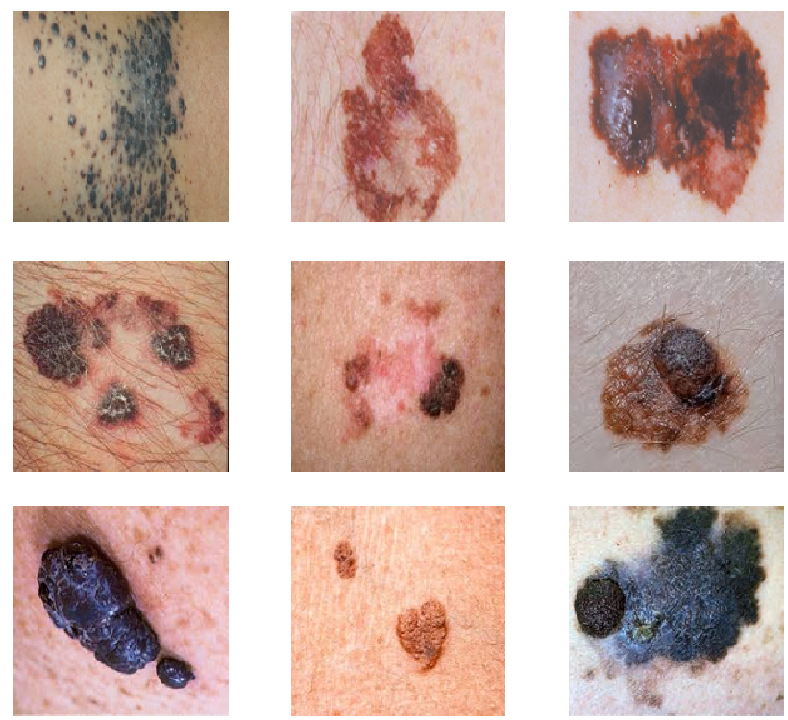

Fig 2. Sample Input Image Dataset

The performance of the results analyzed in terms of accuracy, sensitivity and specificity metrics. These three terms are defined as follows:

\section{Sensitivity}

It defines the proportion of patterns correctly identified as such.

\section{Specificity}

$$
\text { Sensitivity }=\frac{T P}{T P+F N}
$$

It defines the proportion of actual negative patterns which are correctly identified as such.

$$
\text { Specificity }=\frac{T N}{T N+F P}
$$

\section{Accuracy}

It is the probability that the diagnostic test is performed correctly.

$$
\text { Accuracy }=\frac{\mathrm{TP}+\mathrm{TN}}{\mathrm{TP}+\mathrm{TN}+\mathrm{FP}+\mathrm{FN}}
$$

\section{B. Experimental setup}

To evaluate the performance of the system, every skin images in the dataset undergoes image preprocessing techniques. It is used to correct illumination, remove small spots \& noise and enhance the contour and contrast of the images without degrading the lesion features. This can be achieved here by applying filters and contour dilations. PCA in the preprocessing stage used for dimensionality reduction. For better visualization it takes a cloud of data points and rotates it to visualize maximum data variables. After preprocessing the lesion is undergone spatial encoding based feature analysis. Let consider $\mathrm{L}=305$ where $\mathrm{L}$ is the total number of training images. This system recognizes 4 patterns and so take $Y=4$. It uses the sub region size as $\mathrm{L} 3(\mathrm{~N}=5)$ and the window size descriptors into 4880(16*305) clustered features in the BoF codebook. Based on the codebook features Multi-SVM classifies the lesion as homogenous, reticular, globular and multi-component. The classification rate is compared and evaluated with other techniques. This paper also includes sensitivity and specificity measures.

\section{Discussion}

The BoF based Spatial Encoding Algorithm and its descriptors are tested under different levels of sub images denoted as L0(2x2), L1(3x3), L2(4x4) and L3(5x5). Here M and N in the form of MxN denotes MxN dimension of sub image and consider the sliding window size is $2 \times 2$. Let take the SURF feature points as 50(weak), 100 (Strong) and 200(Strong).The dermoscopic images taken for evaluation are of 20x magnification and in JPEG formats. The classes C1, C2, C3 and C4 denotes Homogeneous, Globular, Reticular and Multicomponent patterns. Of the total images of 4 each patterns (Globular, Homogeneous, Reticular, Multicomponent), some of them are clustered lesion. The algorithm was evaluated using leave-one-out approach and considers both color and texture features. 
Since the feature extraction operation depends on various parameters, the algorithm explicitly vary the parameters in order to achieve better results. Initially the whole image is partitioned into $2 \times 2$ sub image and finds SURF feature points for each sub region that forms 4 bags of cluster points. The number of SURF feature points varies as 50 weak points, 100 strong points and 200 strong points. Coding in the algorithm that form a codebook for each clustered region and BoF of sliding window concatenates the featured codebook. The algorithm tested up to $5 \times 5$ dimension of sub image size with varying feature points.

Table I summarizes the results obtained on testing of dermoscopic image database with varying sub image levels and SURF feature points. From the observation, the average melanoma patterns are significantly classified when computing on the set of training images with increasing sub image levels. In particular, reticular and Multi-component patterns tend to produce better classification results due to the presence of pigmented network and associated color combinations. The clustered code book contains more information on feature point variations and its discriminations. In the feature extraction and analysis step, the single vector thus formed with window embedding have represents the distribution of small scale features within the interest point neighborhood. This can also be a reason to have better accuracy for reticular and Multi-component patterns and produce significant results for other patterns too. It is also noted that the algorithm perform almost $98 \%$ accuracy on working with clustered lesions. Finally, sub image was fixed to L3 (5x5) and set the feature point as 200 in order to achieve the trade-off between complexity and size. Table II provides the results of all performance metrics that considered.

TABLE I EXPERIMENTAL RESULTS USING VARIOUS SUB IMAGE LEVELS AND SURF FEATURE POINTS

\begin{tabular}{c|cccc|cccc|ccccc}
\hline & \multicolumn{3}{|c|}{ SURF features(50(weak)) } & \multicolumn{2}{c|}{ SURF Features(100(strong)) } & \multicolumn{3}{c}{ SURF Features(200(strong)) } \\
\hline $\mathbf{L}$ & $\mathrm{C} 1$ & $\mathrm{C} 2$ & $\mathrm{C} 3$ & $\mathrm{C} 4$ & $\mathrm{C} 1$ & $\mathrm{C} 2$ & $\mathrm{C} 3$ & $\mathrm{C} 4$ & $\mathrm{C} 1$ & $\mathrm{C} 2$ & $\mathrm{C} 3$ & $\mathrm{C} 4$ \\
$\mathbf{L 0 ( 2 x 2 )}$ & 80.398 & 81.49 & 82.689 & 84.38 & 82.003 & 83.34 & 84.96 & 86.66 & 86.93 & 85.65 & 90.43 & 91.55 \\
$\mathbf{L 1 ( 3 x 3 )}$ & 82.54 & 83.65 & 78.25 & 87.56 & 84.57 & 85.65 & 88.71 & 88.50 & 88.75 & 87.55 & 92.35 & 93.67 \\
$\mathbf{L 2 ( 4 x 4 )}$ & 85.75 & 85.45 & 79.60 & 89.45 & 88.65 & 87.51 & 90.56 & 92.45 & 90.85 & 89.45 & 94.45 & 95.49 \\
$\mathbf{L 3 ( 5 x 5 )}$ & 89.50 & 87.75 & 90.75 & 92.45 & 91.37 & 89.35 & 93.54 & 94.2 & 92.45 & 94.42 & 96.06 & 97.37 \\
\hline
\end{tabular}

TABLE II OBSERVED AVERAGE QUALITATIVE RESULTS BY THE PROPOSED SYSTEM

\begin{tabular}{cccccc}
\hline Parameters & Globular & Homogeneous & Reticular & Multi-Component & Average Rate(\%) \\
\hline Accuracy & 92.45 & 94.42 & 96.06 & 97.37 & 95.075 \\
Sensitivity & 89.41 & 93.99 & 95.83 & 97.05 & 94.07 \\
Specificity & 93.63 & 94.78 & 96.13 & 97.46 & 95.5 \\
\hline
\end{tabular}

TABLE III COMPARATIVE RESULT OF PROPOSED METHOD WITH EXISTING CLUSTERING TECHNIQUES

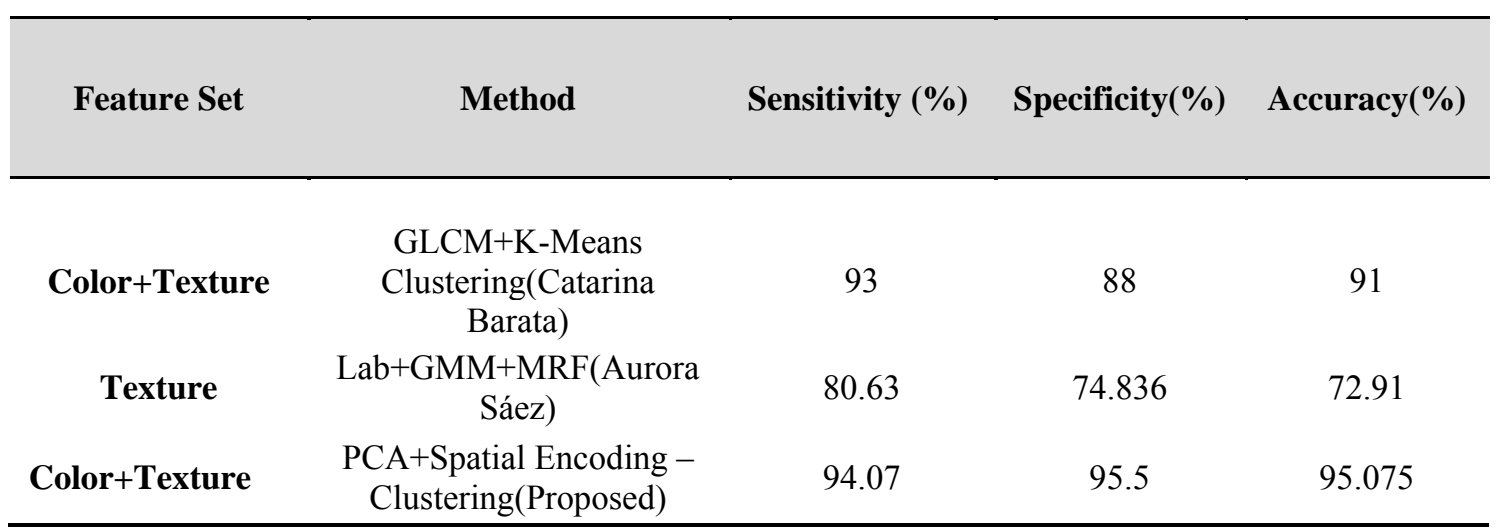


The results thus found shows that the proposed method gives better discriminations among various patterns when compared with other clustering techniques. Table III shows the results of existing clustering techniques and the proposed one. In paper [9] an image is modeled by an MRF on the $1 * \mathrm{a} * \mathrm{~b}$ color space and are divided into 81x81 patch size. The cluster code book contains MRF features with n-dimensional vector per class produces the accuracy as $72.91 \%$ and sensitivity, specificity rates as $80.63 \%$ and $74.836 \%$ respectively. Clustering based feature extraction used in [15] follows color and texture based feature points as melanoma discriminations. In this BoF based technique, key points are of regular grid size $\Delta \mathrm{x} \Delta$ in the image domain. By varying the bins of histogram as $15,16 \ldots 50$, the algorithm produces the accuracy as $91 \%$ and sensitivity, specificity rates as $93 \%$ and $88 \%$ respectively . From all the above discussions, the proposed clustering technique produces substantial improvement on lesion images over other clustering techniques.

\section{CONCLUSION AND FUTURE SCOPE}

The proposed early detection of the lesion pattern recognition system using BoF based Supervised Spatial Encoding of clustered technique provides better results when compared with other existing clustered techniques. Most of the authors focus on the lesion malignancy. But, this pattern recognition system is used to find the melanoma types. Since the Spatial Encoder considers the whole image as lesion, it also recognizes clustered or patched images. Low Level Images using BoF representation provides vector features. Vector features so formed are concatenated and embedded in the codebook of features. Finally, the features on the codebook are classified using multi-SVM classifier. The performance of the existing methods shows that they provide better results for less pattern types and for less data elements. The system works on clustered images also. Scale and rotation variant images are also tested. This system provides $95.075 \%$ accuracy, $94.07 \%$ sensitivity and $95.5 \%$ specificity rates on an average for four classes and the database uses 305 data elements.

This system uses the color and texture features in spatial domain. In future, the system aims to enhance the qualitative rates by considering geometric features. This can achieve by normalizing the feature sets.

\section{REFERENCES}

[1] American Cancer Society, "Skin Cancer: Basal and Squamous Cell" American Cancer Society, Atlanta, Ga .[online].Available:http://www.cancer.org/cancer/skin cancer, 2016.

[2] Nishima Sachdeva, Rohan Gupta, "A Brief Review of classification and segmentation methods for Skin Cancer Images", International Journal of Advanced Research in Electronics and Computer Engineering (IJARECE), Vol.5, Issue 6, June2016.

[3] G. Argenziano, H. P. Soyer, V. D. Giorgio, D. Piccolo, P. Carli, M. Delfino, A. Ferrari, R. Hofmann-Wellenhof, D. Massi, G. Mazzocchetti, M. Scalvenzi, and I. H. Wolf, "Dermoscopy, An Interactive Atlas EDRA Medical Publishing", [online]. Available: http://www.der-moscopy.org, 2000.

[4] S.Suer, S.Kockara, and M.Mete, "An improved border detection in dermoscopy images for density based clustering", BMC bioinformatics.,vol.12, no.10,p.S12, 2011.

[5] Shivangi Jain,Vandana jagtap, Nitin Pise," Skin Cancer Detection Using Diigital Imaging Processing', Intl Journal of scientific Engineering and Research(IJSER), 5I vol..03 Issue 6,pp.138-140, June2015.

[6] H. Pehamberger, A. Steiner, and K. Wolff, "In vivo epiluminescencemicroscopy of pigmented skin lesions. I. Pattern analysis of pigmentedskin lesions," J. Am. Acad. Dermatol., vol. 17, no. 4, pp. 571-583,1987.

[7] G.Rezze, B. De Sa and R. Neves, Dermoscopy: The pattern analysis", Anias Brasileiros Dermatologia, vol.81, no. 3, pp. 261-268, 2006.

[8] Jonna Jaworek-Korjakowska and Pawel Kleczek, "Automatic Classification of Specific Melanocytic Lesions Using Artifical Inttelligence", BioMed Research International volume 2016.

[9] Aurora Saez, Carmen Serrano, Begona Acha, "Model-Based Classification Methods of Global Patterns in Dermoscopic Images", IEEE. Trans on Med..Imaging, Vol.33, no.5, May 2014.

[10] H. Ganster, A. Pinz, E. Wildling, M. Binder, and H. Kittler, “Automated melanoma recognition,” IEEE Trans. Med. Imaging, vol. 20, no. 3, pp. 233-239, Mar. 2001.

[11] M. Elbaum, "Computer-aided melanoma diagnosis," Dermatol. Clin., vol. 20, no. 4, pp. 735-747, Oct. 2002.

[12] P. Rubegni, G. Cevenini, M. Burroni, R. Perotti, G. Dell'Eva, P. Sbano, and C. Miracco, "Automated diagnosis of pigment skin lesions,” Int. J. Cancer, vol. 101, no. 6, pp. 576-580, Oct. 2002.

[13] A. Blum, H. Luedtke, U. Ellwanger, R. Schwabe, G. Rassner, and C. Garbe, "Digital image analysis for diagnosis of cutaneous melanoma. Development of a highly effective computer algorithm based on analysis of 837 melanocytic lesions," Brit. J. Dermatol., vol. 151 , no. 5, pp. 1029- 1038, Nov. 2004.

[14] Yogendra Kumar Jain, Megha Jai , "Comparison between Different Classification methods wth application to skin cancer" , International Journal of Computer Applications vol. 53-No.11, September 2012.

[15] Catarina Barata, Margarida Ruela, Mariana Francisco, Teresa Mendonça, and Jorge S. Marques, " A Bag-of-Features Approach for the classification of Melanomas in dermoscopy Images: The Role of Color and Texture Descriptors", Computer Vision Technigues for the diagnosis of Skin Cancer, IEEE systems journal 2014.

[16] Lucia Ballerini, Robert B.Fisher, Ben Aidridge, Jonathan Rees "A color and textue based hierarchia K-NN approach to the classification of non-melanoma skin, Department of dermatology 2015.

[17] T. Tanaka, S. Torii, I. Kabuta, K. Shimizu, and M. Tanaka, "Pattern classification of nevus with texture analysis," IEE J. Trans. Electr. Electron. Eng., vol. 3, no. 1, pp. 143-150, 2008.

[18] Aurora Saez, Carmen Serrano, Begona Achai, "Model-Based Classification of Global Patterns in Dermoscopic Images", IEEE Trans. on medical imaging, VOL.33, NO.5, May 2014.

[19] H. Iyatomi, M. E. Celebi, G. Schaefer, M. Tanaka, "Automated color calibration method for dermoscopy images," Computerized Medical Imaging and Graphics, 89-98, 2011.

[20] Tuhin Utsab Paul, Pranavesh Banerjee, Anannya Mukherjee and Samir Kr.Bandhyapadhyay, "Technologies in Texture Analysis- A Review", British Journal of Applied Science \& Technology., 13(6): 1-21, 2016.

[21] Dimitriy Bespalov, Yanjun I,Bing Bai,Ali Sokoufandeh, "Large-scale Image Classification Using Supervised Spatial Encoder" IEEE conf on pattern classification (ICPR), Nov.2012. 
[22] Richard Nock, Frank Nielson, "Statistical Region Merging," IEEE Trans on Pattern Analysis., vol. 26,no. 11,Nov 2004.

[23] M.Eme Celebi, Hassan A..Kingravi, Hitoshi lyatomi, Jeongkyu Lee, Y.Alp Aslandogan, William Van Stoecker " Fast and Accurate Border Detection in Dermoscopy Images Using Statistical Region Merging” Proceeding of SPIE Medical Imaging, Feb. 17 - 22. San Diego, CA, USA, 2007.

[24] Felsia Thompson, M.K Jeyakumar, “Vector Based Classification of Dermoscopic Images using SURF”, IJAER, vol 12, no.8, PP.1758$1764,2017$.

[25] Toshiyuki Tanaka, Satoru Torii,Iukei Kabuta,Kunio Shimizu, Masaru Tanaka, "Pattern Classification of Nevus with Texture Analysis", IEEJ Trans vol. 3:, pp:143-150, 2008.

\section{ABOUT THE AUTHOR}

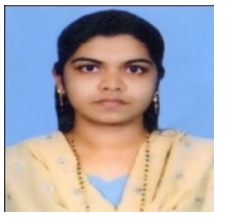

Felsia Thompson is a research scholar at the department of Computer Applications. Noorul Islam Center for Higher Education, Noorul Islam University, Tamilnadu, India. Her research interest is Image Processing.



Dr. M. K. Jeya Kumar is working as a professor in the Department of Computer Applications, Noorul Islam Center for Higher Education, Tamilnadu, India. He has 23 years of teaching experience including 12 years of research experience in the field of Mobile Computing and Image Processing. He published more than 75 peer review research articles and one book chapter. 